\title{
A clinical prediction model for prolonged air leak after pulmonary resection
}

\author{
Adam Attaar, BS, ${ }^{a}$ Daniel G. Winger, MS, ${ }^{b}$ James D. Luketich, MD, ${ }^{a}$ Matthew J. Schuchert, MD, ${ }^{a}$ \\ Inderpal S. Sarkaria, MD, ${ }^{a}$ Neil A. Christie, MD, ${ }^{a}$ and Katie S. Nason, MD, MPH
}

\section{ABSTRACT}

Objective: Prolonged air leak increases costs and worsens outcomes after pulmonary resection. We aimed to develop a clinical prediction tool for prolonged air leak using pretreatment and intraoperative variables.

Methods: Patients who underwent pulmonary resection for lung cancer/nodules (from January 2009 to June 2014) were stratified by prolonged parenchymal air leak ( $>5$ days). Using backward stepwise logistic regression with bootstrap resampling for internal validation, candidate variables were identified and a nomogram risk calculator was developed.

Results: A total of 2317 patients underwent pulmonary resection for lung cancer/ nodules. Prolonged air leak $(8.6 \%, \mathrm{n}=200)$ was associated with significantly longer hospital stay (median 10 vs 4 days; $P<.001$ ). Final model variables associated with increased risk included low percent forced expiratory volume in 1 second, smoking history, bilobectomy, higher annual surgeon caseload, previous chest surgery, Zubrod score $>2$, and interaction terms for right-sided thoracotomy and wedge resection by thoracotomy. Wedge resection, higher body mass index, and unmeasured percent forced expiratory volume in 1 second were protective. Derived nomogram discriminatory accuracy was $76 \%(95 \%$ confidence interval $[\mathrm{CI}], 0.72-0.79)$ and facilitated patient stratification into low-, intermediate- and high-risk groups with monotonic increase in observed prolonged air leaks $(2.0 \%, 8.9 \%$, and $19.2 \%$, respectively; $P<.001)$. Patients at intermediate and high risk were 4.80 times (95\% CI, 2.86-8.07) and 11.86 times (95\% CI, 7.21-19.52) more likely to have prolonged air leak compared with patients at low risk.

Conclusions: Using readily available candidate variables, our nomogram predicts increasing risk of prolonged air leak with good discriminatory ability. Risk stratification can support surgical decision making, and help initiate proactive, patient-specific surgical management. (J Thorac Cardiovasc Surg 2017;153:690-9)

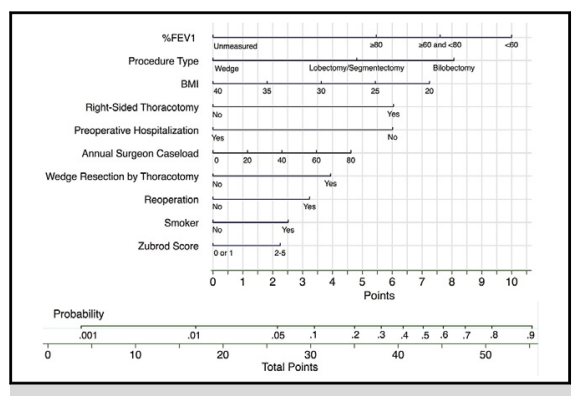

Points for all risk factors are summed to a total score and probability estimate.

\section{Central Message}

Prolonged air leak increases mortality and length of stay after pulmonary resection. Preoperative variables can be combined to predict risk, which may be useful to guide patientspecific management.

\section{Perspective}

We have identified known and new risk factors for prolonged air leak in a large dataset from the United States and developed a prediction nomogram with good discriminatory accuracy. Because prolonged air leak worsens perioperative outcomes, this type of highly accurate risk prediction tool, which uses pre- and intraoperative variables, has the potential to improve patient management and outcomes.

See Editorial Commentary page 700. See Editorial page 688.
When risk factors for undesirable outcomes are identified and used to formulate validated, highly accurate, risk stratification algorithms, allocation of effort and resources

From the a Division of Thoracic and Foregut Surgery, Department of Cardiothoracic Surgery; ${ }^{\mathrm{b}} \mathrm{Clinical}$ and Translational Science Institute, University of Pittsburgh, Pittsburgh, Pa.

This work was supported by The American Association of Thoracic Surgery Summer Intern Scholarship, and Award Numbers K07CA151613 (to K.S.N.) and UL1TR000005 from the National Cancer Institute and National Institutes of Health supported the study. The content is solely the responsibility of the authors, and does not represent the official views of the funding source. to individuals most likely to benefit can be optimized. For thoracic surgeons, prolonged air leak (PAL) after pulmonary resection is one such undesirable outcome. Defined
Read at the 96th Annual Meeting of The American Association for Thoracic Surgery, May 14-18, 2016, Baltimore, Maryland.

Received for publication May 23, 2016; revisions received Sept 15, 2016; accepted for publication Oct 5, 2016; available ahead of print Nov 29, 2016.

Address for reprints: Katie S. Nason, MD, MPH, 5200 Center Ave, Suite 715, Shadyside Medical Building, Pittsburgh, PA 15232 (E-mail: nasonks@upmc.edu). $0022-5223 / \$ 36.00$

Copyright (C) 2016 by The American Association for Thoracic Surgery http://dx.doi.org/10.1016/j.jtcvs.2016.10.003 


\section{Abbreviations and Acronyms \\ CI = confidence interval \\ DLCo = diffusing capacity of carbon monoxide \\ $\% \mathrm{FEV}_{1}=$ percent forced expiratory volume in \\ 1 second \\ PAL $=$ prolonged air leak \\ STSGTSD $=$ Society of Thoracic Surgeons General Thoracic Surgery Database}

Scanning this QR code will take you to supplemental figures, table, video, and appendix for this article.

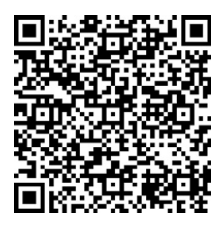

in the Society of Thoracic Surgeon's General Thoracic Surgery Database (STSGTSD) as a parenchymal air leak lasting more than 5 days, PAL complicates $6 \%$ to $18 \%$ of lung resections. ${ }^{1-5}$ PAL is associated with increased cost ${ }^{6}$ increased hospital length of stay, ${ }^{4,5,7}$ and incidence of empyema, ${ }^{6,8,9}$ among other complications. ${ }^{10}$ Often, persistent parenchymal air leak is the only reason for ongoing hospitalization, resulting in eventual discharge with indwelling chest tubes. If patients are unprepared for this eventuality or their care facilities are unable to accommodate patients with chest tubes, additional delays in discharge may be introduced. Therefore, identification of patients at risk for PAL could facilitate proactive patient-specific management.

Three centers, all outside the United States, have published PAL risk stratification models in an effort to improve targeted use of surgical adjuncts. ${ }^{1-3}$ Each propose different criteria for stratifying risk based on independent risk factors for PAL, including percent forced expiratory volume in 1 second $\left(\% \mathrm{FEV}_{1}\right)$, body mass index (BMI), and pleural adhesions, among other variables. Study design limitations, differences in how PAL was defined, how patients were selected, and lack of external prospective validation have inhibited wide clinical application. It is also unclear whether risk factors identified in these studies are generalizable and useful for predicting risk for PAL in patients in the United States.

Our study sought to develop a clinical prediction tool for PAL after pulmonary resection using pretreatment and intraoperative variables in a large patient dataset from a single center in the United States. We hypothesized that standard, readily available predictors could be used to stratify patients into risk classes associated with increasing PAL risk.

\section{METHODS}

\section{Patient Population and Data Definitions}

Data for all patients were collected at our institution using variables defined by the STSGTSD (http://www.sts.org/national-database). Data variables were defined using versions 2.081 and 2.2 ; data were abstracted by trained data collectors within 4 to 6 weeks after operation for real-time quality monitoring and national benchmarking via biannual data submission to the STSGTSD National Data Center. Our institutional review board gave approval for use of this data for the current study.

Pulmonary resection was performed $(\mathrm{n}=2522$; from January 1,2009 to 30 June, 2014) at 8 hospital sites for malignant and benign lung tumors or nodules using International Classification of Diseases, Ninth Revision ${ }^{11}$ diagnosis codes $(197.0,212.3,162.2,162.3,162.4,162.5,162.9$, and 518.89), excluding pneumonectomy, and extended chest wall/diaphragm resections. We did not include volume reduction, bullectomy, or lung biopsy for interstitial lung disease because of the significantly higher rate of PAL and differences in underlying risk factors in these populations, which would have heavily influenced the model and reduced discriminatory accuracy for PAL in patients with lung nodules/cancer. Database variables included patient demographics, preoperative evaluation, surgical procedures, cancer staging, and postoperative events. Approach to operation was captured, but granular operative details were not available (eg, intraoperative adhesiolysis, intraoperative sealant use, pleural tents, and so forth).

We excluded 59 patients who had undergone sleeve lobectomy to minimize confounding parenchymal and anastomotic air leaks; 32 patients from 2 hospital sites who did not submit patients to the database over the entire study time frame; and 5 patients who died by day 5 or before (PAL definition). Status of air leak for patients dying by day 5 or before is not available in the dataset. Multiple lung resections were noted in 111 patients; data for the most recent surgery date were used and previous encounter(s) excluded (Figure E1).

\section{Outcomes}

PAL, the dependent variable in our model, was defined as an air leak that persisted for more than 5 days postoperatively. We compared in-hospital mortality, length of hospital stay, and cumulative incidence of hospital discharge by postoperative day (mortality treated as a competing risk), ${ }^{12}$ between groups.

\section{Development of the Logistic Regression Model}

We compared pre- and intraoperative variables with chi-square test or Fisher exact test for categorical variables expressed as frequencies, and Student $t$ test or Wilcoxon rank sum test for continuous variables expressed as mean \pm 1 standard deviation or median/interquartile range using Stata/ SE 14.1 (Stata Statistical Software, 2015; Stata Corporation, College Station, Tex). Unless otherwise indicated, $P$ values were 2-tailed; statistical significance defined by $P<.05$.

Bivariable testing $(P<.15)$, clinical judgment, and literature review guided variable selection for the initial multivariable model. We categorized $\% \mathrm{FEV}_{1}$ based on clinical relevance: $<60 \%$ considered moderate to severe obstruction, $\geq 60 \%$ and $<80 \%$ mild obstruction, $\geq 80 \%$ borderline to normal range. We placed the $12.8 \%$ of patients who did not undergo preoperative pulmonary function testing, and were therefore missing on $\% \mathrm{FEV}_{1}$, in a separate category because most had benign or secondary metastatic lung tumors $(86.5 \%)$ removed with wedge resection $(88.9 \%)$, suggesting that the data were systematically missing (ie, pulmonary function testing was not performed intentionally). We excluded the diffusing capacity of carbon monoxide (DLCo) because of the high level of nonrandom missingness. We included sex and steroids variables despite $P>.15$, given their significance in previous work. To assess collinearity, we assessed variance inflation factors and ensured that all were less than 2 (indicating little $(>1<2)$ to no $(=1)$ correlation between variables). 


\begin{tabular}{|c|c|c|c|c|}
\hline \multirow[b]{2}{*}{ Variables } & \multirow[b]{2}{*}{$\begin{array}{c}\text { Total } \\
\mathbf{n}=\mathbf{2 3 1 7}\end{array}$} & \multicolumn{2}{|c|}{ PAL } & \multirow[b]{2}{*}{$\boldsymbol{P}$} \\
\hline & & $\begin{array}{c}\text { No } \\
\mathbf{n}=\mathbf{2 1 1 7} \\
\end{array}$ & $\begin{array}{c}\text { Yes } \\
\mathbf{n}=\mathbf{2 0 0}\end{array}$ & \\
\hline \multicolumn{5}{|l|}{ Demographics } \\
\hline Age $($ mean \pm SD) & $65 \pm 12$ & $65 \pm 12$ & $67 \pm 11$ & .013 \\
\hline BMI, $\mathrm{kg} / \mathrm{m}^{2}($ mean $\pm \mathrm{SD})$ & $28 \pm 7$ & $28 \pm 7$ & $26 \pm 5$ & $<.001$ \\
\hline \multicolumn{5}{|l|}{ Sex } \\
\hline Female & $1296(56)$ & $1193(56)$ & $103(51)$ & .186 \\
\hline Male & $1021(44)$ & $924(44)$ & 97 (49) & \\
\hline \multicolumn{5}{|l|}{ Race } \\
\hline White & $2168(94)$ & $1975(93)$ & $193(96)$ & $.288^{*}$ \\
\hline Black & $119(5)$ & $113(5)$ & $6(3)$ & \\
\hline Other & $30(1)$ & $29(1)$ & $1(1)$ & \\
\hline \multicolumn{5}{|l|}{ Treatment variables } \\
\hline \multicolumn{5}{|l|}{ Surgery year } \\
\hline 2009/10 & 857 (37) & 777 (37) & $80(40)$ & .615 \\
\hline $2011 / 12$ & $886(38)$ & $815(38)$ & $71(35)$ & \\
\hline $2013 / 14$ & $574(25)$ & $525(25)$ & $49(25)$ & \\
\hline \multicolumn{5}{|l|}{ Hospital type } \\
\hline Academic & $1125(49)$ & $1030(49)$ & $95(48)$ & .755 \\
\hline Community & $1192(51)$ & $1087(51)$ & $105(52)$ & \\
\hline Annual surgeon caseload, median [IQR $] \dagger$ & $53[34-74]$ & $53[34-74]$ & $64[36-74]$ & $<.001 \ddagger$ \\
\hline \multicolumn{5}{|l|}{ Disease category } \\
\hline Malignant cancer & $1441(62)$ & $1290(61)$ & $151(76)$ & $<.001$ \\
\hline Benign/infection & $367(16)$ & $346(16)$ & $21(10)$ & \\
\hline Metastatic tumor & $509(22)$ & $481(23)$ & $28(14)$ & \\
\hline \multicolumn{5}{|l|}{ Procedure type } \\
\hline Lobe/segment & $1500(65)$ & $1339(63)$ & $161(81)$ & $<.001^{*}$ \\
\hline Wedge resection & $780(34)$ & $752(36)$ & $28(14)$ & \\
\hline Bilobectomy & $37(1)$ & $26(1)$ & $11(5)$ & \\
\hline \multicolumn{5}{|l|}{ Comorbidities } \\
\hline \multicolumn{5}{|l|}{ Smoking history } \\
\hline Never smoker & $684(30)$ & $655(31)$ & $29(14)$ & $<.001$ \\
\hline Past smoker & $1180(50)$ & $1058(50)$ & $122(61)$ & \\
\hline Current smoker & $453(20)$ & 404 (19) & $49(25)$ & \\
\hline \multicolumn{5}{|l|}{ Zubrod score } \\
\hline 0 & $163(7)$ & $153(7)$ & $10(5)$ & .012 \\
\hline 1 & $1756(76)$ & $1615(77)$ & $141(71)$ & \\
\hline $2-5$ & $390(17)$ & $342(16)$ & $48(24)$ & \\
\hline \multicolumn{5}{|l|}{ ASA classification } \\
\hline $\mathrm{I} / \mathrm{II}$ & $277(12)$ & $263(13)$ & $14(7)$ & .077 \\
\hline III & $1781(77)$ & $1618(76)$ & $163(82)$ & \\
\hline IV & $259(11)$ & $236(11)$ & $23(11)$ & \\
\hline \multicolumn{5}{|l|}{ Weight loss } \\
\hline$\leq 2 \mathrm{~kg}$ & $1985(86)$ & $1825(86)$ & $160(80)$ & .527 \\
\hline$>2 \mathrm{~kg}$ & $113(5)$ & $102(5)$ & $11(5)$ & \\
\hline Missing & $219(9)$ & $190(9)$ & $29(15)$ & \\
\hline Hypertension & $1297(56)$ & $1192(56)$ & $105(53)$ & .300 \\
\hline Congestive heart failure ( $24 \%$ missing) & $76(4)$ & $67(4)$ & $9(6)$ & .234 \\
\hline Peripheral vascular disease & $225(10)$ & $199(10)$ & $26(13)$ & .101 \\
\hline Interstitial fibrosis & $27(1)$ & $23(1)$ & $4(2)$ & $.286^{*}$ \\
\hline Diabetes & 419 (18) & $392(19)$ & $27(14)$ & .077 \\
\hline COPD & $771(33)$ & $672(32)$ & $99(50)$ & $<.001$ \\
\hline Cerebrovascular disease & $141(6)$ & $127(6)$ & $14(7)$ & .574 \\
\hline Preoperative chemotherapy & $470(20)$ & $433(21)$ & $37(19)$ & .505 \\
\hline Preoperative radiation therapy & $314(14)$ & $282(13)$ & $32(16)$ & .294 \\
\hline
\end{tabular}




\begin{tabular}{|c|c|c|c|c|}
\hline \multirow[b]{2}{*}{ Variables } & \multirow[b]{2}{*}{$\begin{array}{c}\text { Total } \\
\mathbf{n}=\mathbf{2 3 1 7}\end{array}$} & \multicolumn{2}{|c|}{ PAL } & \multirow[b]{2}{*}{$\boldsymbol{P}$} \\
\hline & & $\begin{array}{c}\text { No } \\
\mathbf{n}=\mathbf{2 1 1 7}\end{array}$ & $\begin{array}{c}\text { Yes } \\
\mathbf{n}=\mathbf{2 0 0}\end{array}$ & \\
\hline Steroids & $127(5)$ & $112(5)$ & $15(8)$ & .190 \\
\hline Previous cardiothoracic surgery & $512(22)$ & $455(22)$ & $57(29)$ & .024 \\
\hline Reoperation $\S$ & $289(13)$ & $249(12)$ & $40(20)$ & .001 \\
\hline \multicolumn{5}{|l|}{ Laboratory } \\
\hline $\mathrm{FEV}_{1}, \%$ predicted $(12.8 \%$ missing; mean $\pm \mathrm{SD})$ & $83 \pm 22$ & $84 \pm 21$ & $76 \pm 24$ & $<.001$ \\
\hline \multicolumn{5}{|l|}{$\mathrm{FEV}_{1}, \%$ predicted } \\
\hline$\geq 80$ & $1173(51)$ & $1089(52)$ & $84(42)$ & \multirow[t]{4}{*}{$<.001$} \\
\hline$<60$ & $561(24)$ & $496(23)$ & $65(33)$ & \\
\hline$\geq 60$ and $<80$ & $285(12)$ & $238(11)$ & $47(23)$ & \\
\hline Unmeasured & $297(13)$ & $293(14)$ & $4(2)$ & \\
\hline DLCo, $\%$ predicted $(28 \%$ missing; mean $\pm \mathrm{SD})$ & $71 \pm 23$ & $72 \pm 23$ & $66 \pm 24$ & .002 \\
\hline Last hemoglobin $($ mean $\pm \mathrm{SD})$ & $12.1 \pm 1.7$ & $12.2 \pm 1.7$ & $12.0 \pm 1.7$ & .152 \\
\hline Last creatinine, median [IQR] & $0.85[0.7-1]$ & $0.87[0.7-1]$ & $0.80[0.7-1]$ & .628 \\
\hline \multicolumn{5}{|l|}{ Operative details } \\
\hline \multicolumn{5}{|l|}{ Status } \\
\hline Elective & $2214(96)$ & $2018(96)$ & $196(98)$ & \multirow[t]{2}{*}{.145} \\
\hline Urgent/emergent & $90(4)$ & $86(4)$ & $4(2)$ & \\
\hline \multicolumn{5}{|l|}{ Preoperative hospitalization } \\
\hline$<1 \mathrm{~d}$ & $2157(93)$ & $1963(93)$ & $194(97)$ & \multirow[t]{2}{*}{.025} \\
\hline$\geq 1 \mathrm{~d}$ & $158(7)$ & $152(7)$ & $6(3)$ & \\
\hline \multicolumn{5}{|l|}{ Laterality } \\
\hline Left & $915(40)$ & $854(40)$ & $61(30)$ & \multirow[t]{3}{*}{.004} \\
\hline Right & $1377(59)$ & $1238(59)$ & $139(70)$ & \\
\hline Missing & $25(1)$ & $25(1)$ & $0(0)$ & \\
\hline Robot-assisted surgery & $82(3)$ & $79(4)$ & $3(2)$ & .102 \\
\hline \multicolumn{5}{|l|}{ Surgical approach } \\
\hline Thoracotomy & $603(26)$ & $515(24)$ & $88(44)$ & \multirow[t]{2}{*}{$<.001$} \\
\hline VATS & $1714(74)$ & $1602(76)$ & $112(56)$ & \\
\hline OR blood transfusion & $94(4)$ & $84(4)$ & $10(5)$ & .487 \\
\hline \multicolumn{5}{|l|}{ Outcomes } \\
\hline Hospital stay, median [IQR] & $4[3-7]$ & $4[3-6]$ & $10[8-14]$ & $<.001$ \\
\hline Hospital death & $29(1)$ & $23(1)$ & $6(3)$ & .034 \\
\hline
\end{tabular}

Values n (\%), and $<1 \%$ missing unless indicated otherwise. SD, Standard deviation; BMI, body mass index; IQR, interquartile range; ASA, American Association of Anesthesiology; $C O P D$, chronic obstructive pulmonary disease; $\% F E V_{l}$, percent forced expiratory volume in 1 second; $D L C O$, diffusing capacity of carbon monoxide; $V A T S$, video-assisted thoracoscopic surgery; $O R$, operating room. *Fisher exact test. †Average annual cases by patient's operating surgeon in study time period. $\ddagger$ Wilcoxon rank sum test. $\S$ Cardiac or thoracic reoperation that affects operative field.

To form a parsimonious model, we used backward stepwise variable selection with likelihood ratio test $P$ value stopping rules of .15 (to enter model) and .10 (to remain in model); PAL was the dependent variable. Bootstrap resampling was used to assess how well variables predict occurrence of PAL outside the original sample. ${ }^{8,13}$ We repeated the stepwise algorithm on 1000 resamples drawn randomly with replacement and equal to $100 \%$ size of the original. We considered variables selected in more than $50 \%$ of 1000 repetitions for the reduced model and tested plausible 2-way interactions.

\section{Development of the PAL Nomogram}

Using the Stata program nomolog, ${ }^{14}$ we assigned points to predictors using proportional rescaling of the logistic equation regression coefficients. We first forced positive coefficients with negative regression coefficients by subtracting all categories of the variable by the category with the most negative coefficient. We then multiplied all coefficients by the scaling factor, $\mathrm{F}$ ( $\mathrm{F}=10 / \alpha_{\mathrm{i}}$, where $\alpha_{\mathrm{i}}$ is the largest model coefficient) to convert coefficients into point values. We calculated PAL probability based on total points $\left(1 /\left[1+\exp \left(-\left[\mathrm{F} \times \alpha_{0}+\mathrm{TP}\right] / \mathrm{F}\right)\right]\right)$, where $\alpha_{0}$ is the regression equation constant (adjusted accordingly from variables forced positive), and TP is the sum of points across all predictors. We derived a 3-level risk table based on the nomogram point scoring system (cutoffs chosen to maximize $\mathrm{C}$ statistics), and classified each patient into a risk class using their total point score (rounded to the nearest 0.5 ). We calculated observed and predicted frequencies, odds ratios, and $95 \%$ confidence intervals (CIs) for each risk class.

\section{Model Performance}

We used calibration and discrimination to assess model predictive accuracy. ${ }^{15}$ For calibration, we plotted observed and predicted rates of PAL by deciles of risk, and assessed goodness of fit (Hosmer-Lemeshow test). We calculated discriminatory accuracy (C statistic) for the regression equation, nomogram, and risk table. Using a classification probability cutoff of 0.11 (the average PAL rate in 5 large PAL studies ${ }^{1-5}$ combined with ours), we calculated correct classification rates, and positive and negative predictive values. We used bootstrap resampling to adjust the 


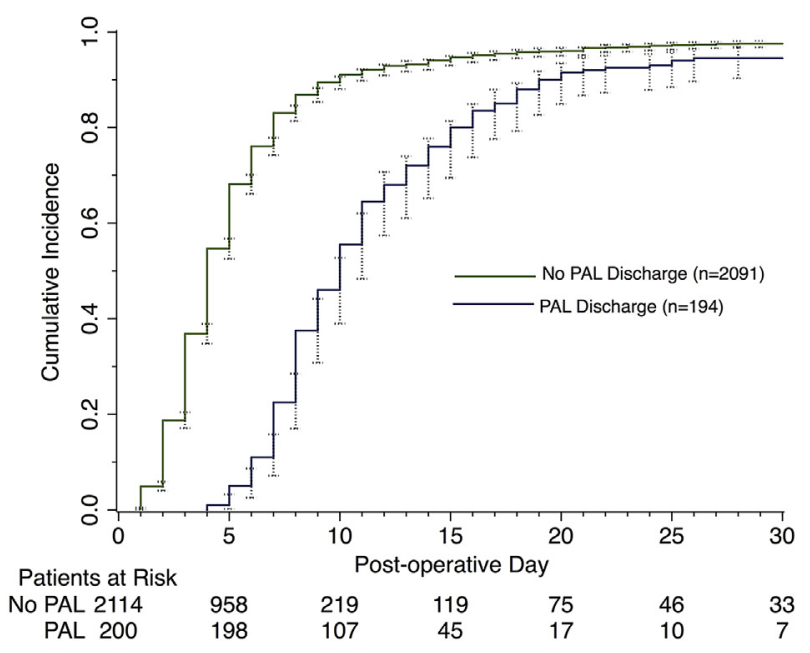

FIGURE 1. Stratified cumulative incidence of hospital discharge by postoperative day (95\% CI indicated by black-capped spikes). PAL, Prolonged air leak.

logistic C statistic for overoptimism or overfitting. ${ }^{15,16}$ We repeated the original stepwise multivariable selection with the addition of identified interaction effects in 500 resamples. In each repetition, we calculated the difference between the $\mathrm{C}$ statistic in the resample and the original sample for the selected model. To adjust for optimism, we subtracted the average difference from the $\mathrm{C}$ statistic of the final model in the original sample.

\section{Surgical Caseload Effect}

We used a funnel plot to explore the relationship between total surgeon caseload and PAL rate, ${ }^{17}$ including control limits (95\% and $99 \%$ CIs) around the average PAL rate in our study. Because unadjusted case volume does not account for variations in patient populations between surgeons, we developed risk-adjusted models for surgeon annual caseload effect, adjusting for case mix and clustering (see Appendix 1).

\section{RESULTS}

A total of 2317 patients met the inclusion criteria for the bivariable analysis and 2273 patients for the multivariable analysis. Incidence of PAL was 8.6\% (200/2317). Most of the operations were video-assisted thoracoscopic (VATS) lobectomy/segmentectomy for primary lung cancer (Table 1). PAL significantly prolonged median length of hospital stay (10 vs 4 days, $P<.001$ ); by day 5 and 10 , respectively, only $1 \%$ and $47 \%$ of patients with PAL had been discharged compared with $54 \%$ and $90 \%$ of patients without PAL (Figure 1). Patients with PAL had a higher rate of in-hospital death $(3.0 \%$ vs $1.1 \%, P=.034$; Table 1$)$.

\section{Development of the Prediction Model}

Patients with PAL were more likely to be older, male, have a lower BMI, previous smoking history, peripheral vascular disease, chronic obstructive pulmonary disease, previous steroid use, higher Zubrod score, and lower \% $\mathrm{FEV}_{1}$. They were less likely to have diabetes or to be hospitalized before surgery. Patients with PAL were more likely to have primary lung cancer (vs benign or metastatic tumors), undergo lobectomy/segmentectomy or bilobectomy (vs wedge resection), have right-sided resection, undergo thoracotomy, and be operated on by a surgeon with higher annual caseloads (Table 1). After backward stepwise logistic regression with bootstrap resampling for reliability, 10 variables comprising 13 separate coefficients were chosen in more than $50 \%$ of resamples

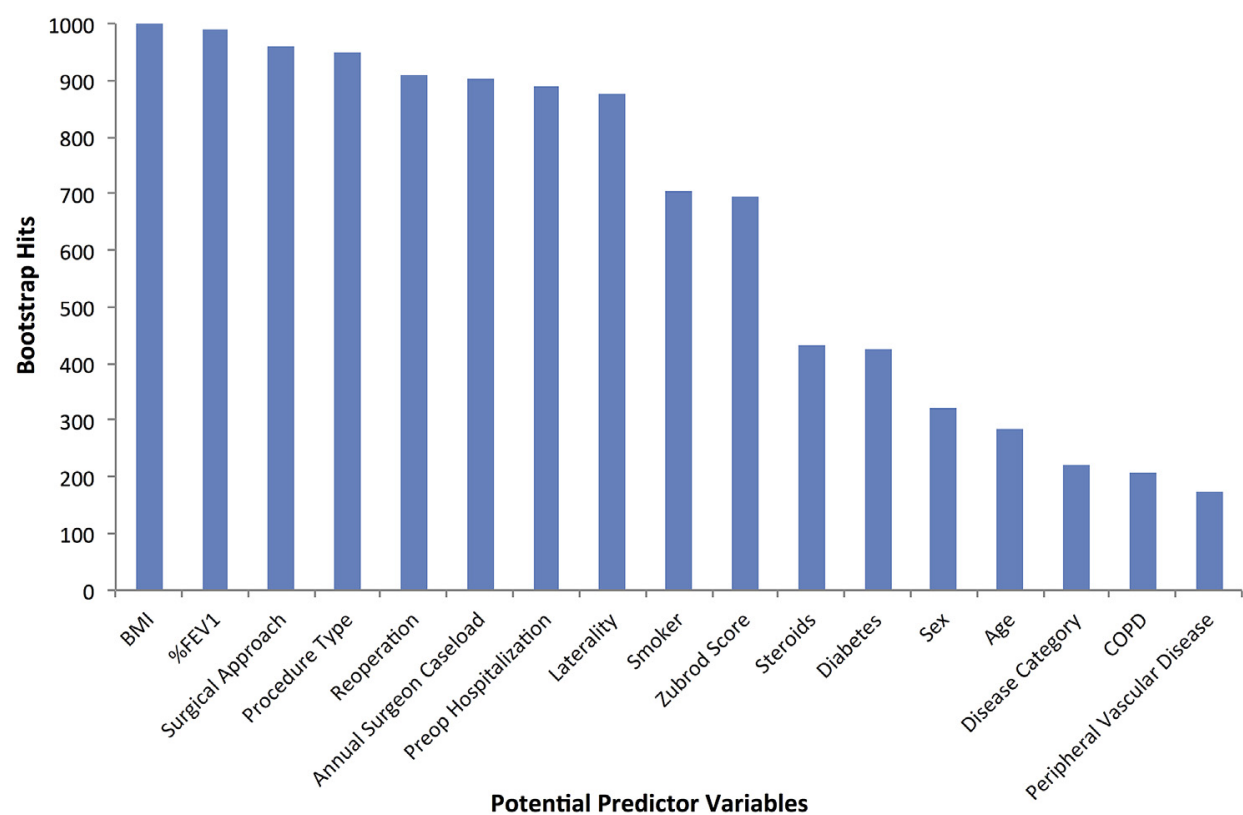

FIGURE 2. Bootstrap reliability of variables associated with prolonged air leak. Variables selected $>50 \%$ of the time were considered for forming the final model. BMI, Body mass index; \%FEVI, percent forced expiratory volume in 1 second; $C O P D$, chronic obstructive pulmonary disease. 
and included in the final model (Figure 2). Among all variables excluded in stepwise variable selection, or on bivariable testing ( $P$ values between .15 and .30 ), none except hypertension were found to have adjusted $P<.10$ or change regression coefficients by more than $10 \%$ with (re)inclusion in the final model. We incorporated the significant interaction effect "right-sided thoracotomy" (between surgical approach and laterality) and "wedge resection by thoracotomy" (between surgical approach and wedge resection) for their regression coefficient adjustment of the individual variables. The final regression model included $\mathrm{BMI}, \% \mathrm{FEV}_{1}$, annual surgeon caseload, smoking, Zubrod score, preoperative hospitalization, reoperation, procedure type, laterality, surgical approach, right-sided thoracotomy, and wedge resection by thoracotomy (Table 2).

\section{Categorizing Risk using the Nomogram}

Using the regression coefficients and intercept from the final model (Table 2), we created a nomogram to calculate the probability of PAL (Figure 3,A). For the interaction effects "right-sided thoracotomy" and "wedge resection by thoracotomy," the main effect variables laterality (right-sided, 0.2 patients) and surgical approach (VATS, 1.6 patients) had small point values and are not depicted in the nomogram. We calculated a nomogram point score for each patient, and categorized patients into low-, intermediate-, and high-risk groups (Table 3). Compared with the lowest-risk group, patients in the intermediaterisk group were more than 5 times more likely to have a PAL, while the high-risk group was more than 12 times more likely (Table 3). The observed rates of PAL were $2.0 \%, 8.8 \%$, and $19.3 \%$ (nonparametric test of trend $P<.001)$, which matched closely with the predicted rates according to the model. A more generalizable nomogram identically derived except for removal of our institution-specific annual surgeon caseload variable was also created (Figure 3,B).

\section{Predictive Performance of the Model and Nomogram}

Discriminatory accuracy of the final regression model was $75.9 \%$ (C statistic, 0.759; 95\% CI, 0.725-0.792). The

TABLE 2. Analysis of potential predictors for prolonged air leak

\begin{tabular}{|c|c|c|c|c|c|c|}
\hline \multirow[b]{2}{*}{ Variable } & \multicolumn{2}{|c|}{ Unadjusted } & \multicolumn{4}{|c|}{ Adjusted } \\
\hline & Odds ratio & $\boldsymbol{P}$ & Odds ratio & $95 \% \mathrm{CI}$ & Coefficient & $\boldsymbol{P}$ \\
\hline Intercept* & & & 0.043 & $0.022-0.086$ & -3.137 & \\
\hline Age (per 1 y increase) & 1.017 & .013 & & & & \\
\hline $\operatorname{BMI}\left(\mathrm{kg} / \mathrm{m}^{2}\right)$ & 0.936 & $<.001$ & 0.938 & $0.912-0.964$ & -0.064 & $<.001$ \\
\hline Sex (female) & 0.822 & .186 & & & & \\
\hline Smoker (vs nonsmoker) & 2.642 & $<.001$ & 1.560 & $1.013-2.402$ & 0.445 & .043 \\
\hline Zubrod score $2-5$ (vs 0 or 1$)$ & 1.643 & .004 & 1.489 & $1.015-2.184$ & 0.398 & .042 \\
\hline Diabetes & 0.686 & .077 & & & & \\
\hline Steroids & 1.451 & .190 & & & & \\
\hline Malignant cancer & 1.665 & .030 & & & & \\
\hline COPD & 2.106 & $<.001$ & & & & \\
\hline Peripheral vascular disease & 1.431 & .101 & & & & \\
\hline Reoperation $\dagger$ & 1.877 & .001 & 1.771 & $1.183-2.651$ & 0.572 & .005 \\
\hline FEV $1, \%$ predicted & & $<.001$ & & & & \\
\hline$\geq 80$ & Ref. & & Ref. & & & \\
\hline$\geq 60$ and $<80$ & 1.699 & & 1.458 & $1.016-2.093$ & 0.377 & .041 \\
\hline$<60$ & 2.560 & & 2.228 & $1.467-3.384$ & 0.801 & $<.001$ \\
\hline Unmeasured & 0.177 & & 0.380 & $0.132-1.096$ & -0.967 & .073 \\
\hline Preoperative hospitalization $\geq 1 \mathrm{~d}$ & 0.867 & .025 & 0.345 & $0.143-0.833$ & -1.063 & .018 \\
\hline Annual surgeon caseload $\ddagger$ & 1.014 & $<.001$ & 1.010 & $1.003-1.018$ & 0.010 & .009 \\
\hline Resection type & & $<.001$ & & & & \\
\hline Lobe/segment & Ref. & & Ref. & & & \\
\hline Wedge resection & 0.310 & & 0.427 & $0.249-0.731$ & -0.851 & .002 \\
\hline Bilobectomy & 3.519 & & 1.778 & $0.793-3.985$ & 0.575 & .162 \\
\hline Right-sided resection (vs left) & 1.571 & .004 & 1.045 & $0.697-1.566$ & 0.044 & .832 \\
\hline Thoracotomy (vs VATS) & 2.444 & $<.001$ & 0.757 & $0.410-1.398$ & -0.278 & .370 \\
\hline \multicolumn{7}{|l|}{ Interaction terms added to final model } \\
\hline Right-sided thoracotomy & & & 2.910 & $1.430-5.924$ & 1.068 & .003 \\
\hline Wedge resection by thoracotomy & & & 2.006 & $0.783-5.140$ & 0.696 & .147 \\
\hline
\end{tabular}




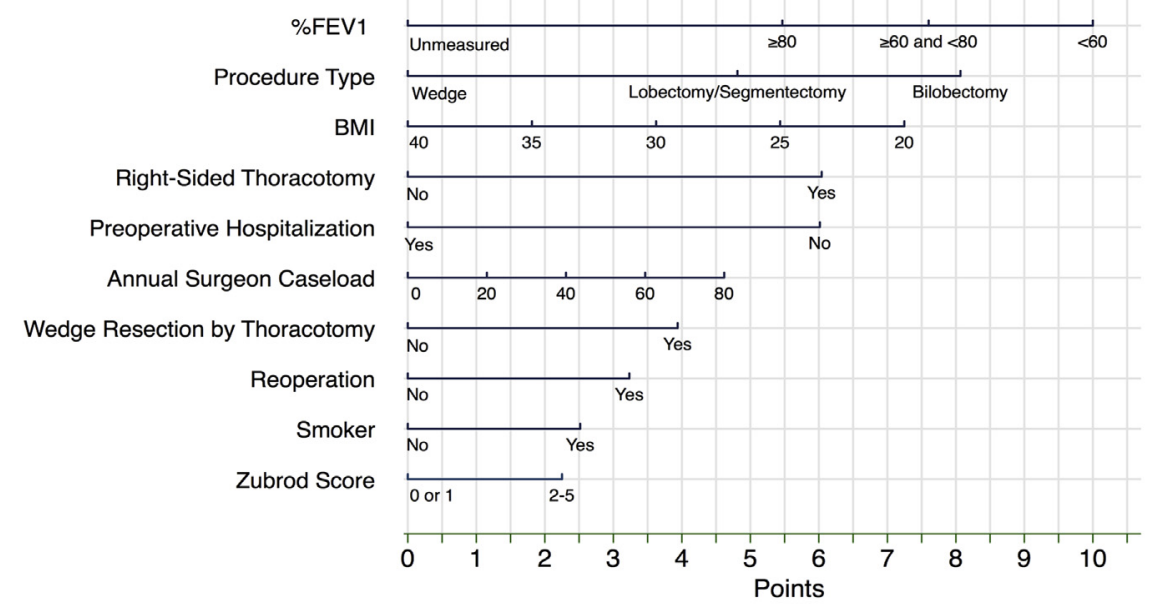

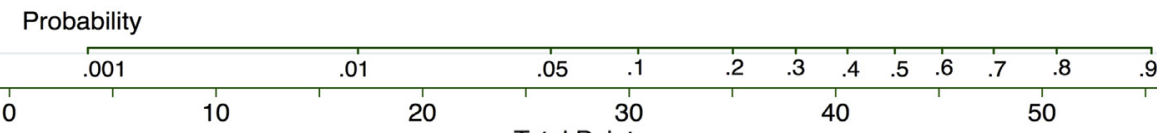

A

Total Points

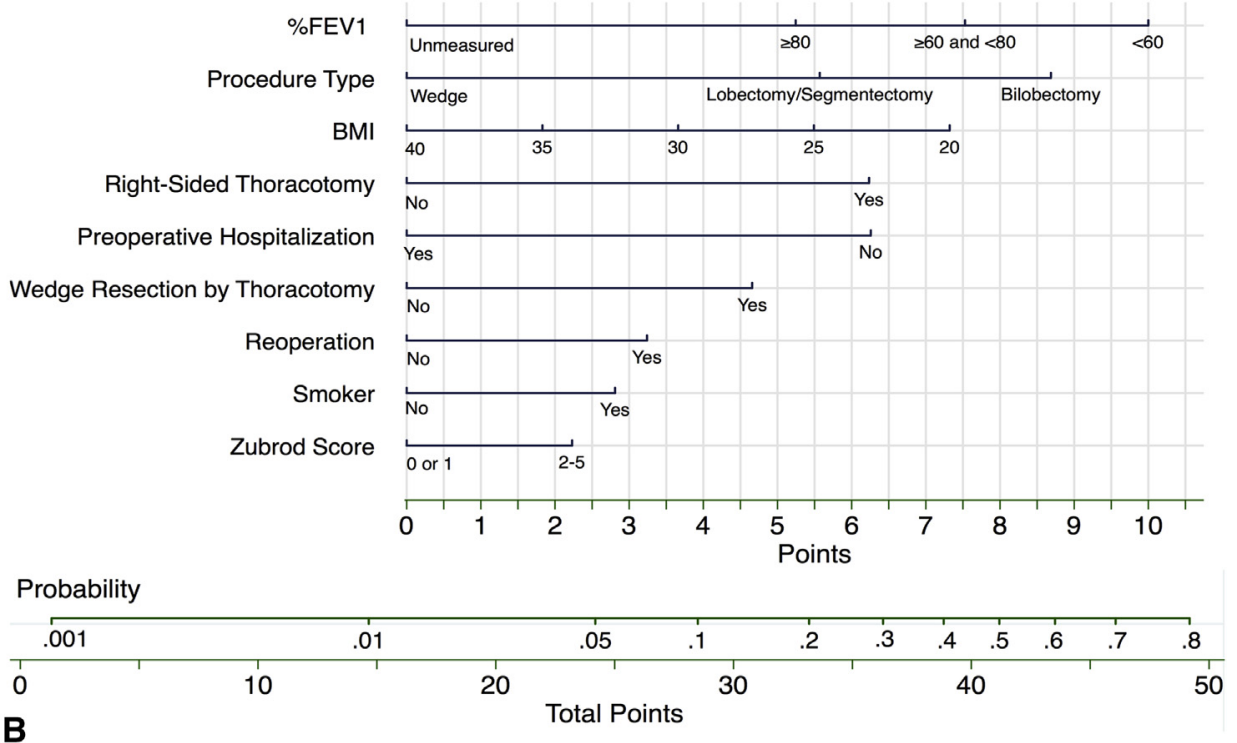

FIGURE 3. Nomogram for probability of prolonged air leak $(P A L)$. A, To calculate the probability of prolonged air leak, sum points over all variables to a total point score with its corresponding probability. Example: a smoker ( 2.5 points $)$ with a body mass index $(B M I)=32$ ( 3 points), percent forced expiratory volume in 1 second $(\% F E V 1)=65$ ( 7.5 points $)$, and Zubrod score $=1$ ( 0 points $)$ without previous chest operation $(0$ points $)$ or preoperative hospitalization $\geq 1$ ( 6 points) is having a right-sided open ( 6 points) lobectomy ( 5 points) by a surgeon who has an annual caseload of 50 ( 3 points). Total points $=33$. Probability of PAL is around $15 \%$. B, We removed our institution-specific variable annual surgeon caseload to create a more generalizable model that had a C statistic $=0.755(95 \% \mathrm{CI}, 0.722-0.788)$

Hosmer-Lemeshow goodness of fit test was nonsignificant $(P=.735)$, indicating good model fit for the data, and the calibration slope was 1.000 with an intercept of 0.000 (showing perfect model calibration). After adjusting for optimism using bootstrap resampling, discriminatory accuracy was $73.8 \%$. The model correctly classifies $74.9 \%$ of patients with a positive predictive value of $19.4 \%$ and a negative predictive value of $95.2 \%$ when calculated using a classification probability cutoff of 0.11 .
The discriminatory accuracy was $75.7 \%$ for the nomogram (C statistic, $0.762 ; 95 \%$ CI, 0.724-0.790), and $72.9 \%$ for the risk table after rounding to the nearest 0.5 for point calculation (C statistic, 0.729; 95\% CI, 0.698-0.760), respectively.

\section{Surgical Volume Effect on PAL Incidence}

To determine the relationship between surgeon volume and PAL incidence, we assessed funnel plots with 
TABLE 3. Classification of PAL

\begin{tabular}{lcccc}
\hline Risk class (score) & PAL incidence $(\mathbf{n})$ & Observed frequency $(\%)$ & Predicted frequency logistic model (\%) & Odds ratio (95\% CI) \\
\hline Low $(\leq 25)$ & 938 & 2.0 & 2.5 & Ref \\
Intermediate $(26-29)$ & 747 & 8.8 & 7.7 & $4.69(2.79-7.88)$ \\
High $(\geq 30)$ & 588 & 19.2 & 19.9 & $11.51(6.99-18.94)$ \\
\hline
\end{tabular}

CI, confidence interval.

calculated control limits (Figure 4). Surgeons with higher annual caseload, on average, have higher PAL incidence (linear correlation 0.38). Data points falling inside control limits are consistent with common cause variation or expected variability in patient population or surgical management. All surgeons lie within the $99 \%$ control limit, essentially excluding special cause or unexpected variation, such as inferior (or superior) surgical technique or management practices. After risk adjustment for case mix (using available Society of Thoracic Surgeons data) and clustering of PAL among surgeon, annual surgeon caseload remained a significant predictor (Table E1 and Figure E2).

\section{DISCUSSION}

Using data from a large, United States-based patient dataset, our study tested the hypothesis that standard, readily available predictors could be used to stratify patients into risk classes associated with increasing risk of PAL. See the narration of key study findings in Video 1. Our analysis identified 10 risk factors for PAL comprising a total of 13 categories with 2 interaction effects and resulted in a parsimonious, reliable, and accurate clinical prediction model for PAL after pulmonary resection. The model coefficients were used to produce a nomogram, which generated probability estimates for PAL and risk classification of patients into low-, intermediate-, and high-risk groups. Importantly, these risk groups showed monotonic increase in the rate of observed PAL, indicating clear differences in risk between

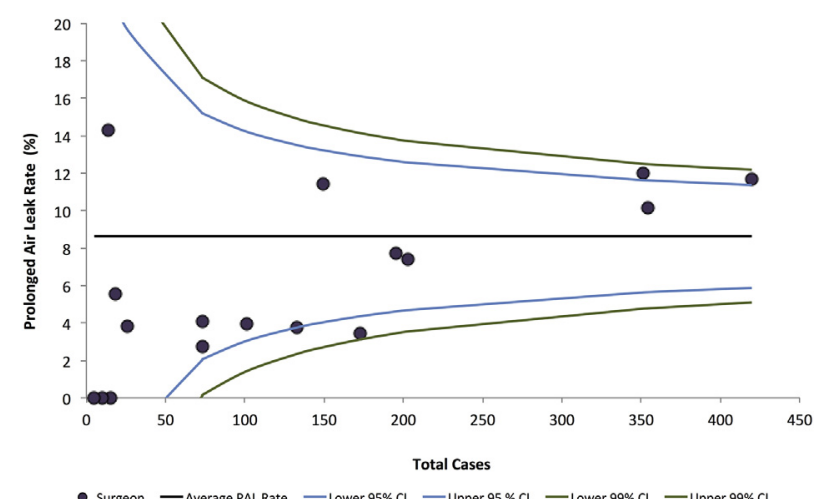

FIGURE 4. Funnel plot of prolonged air leak $(P A L)$ rate of operating surgeons by case volume. Control limits $(95 \%$ and $99 \%)$ were derived as follows: $(\theta \pm z \times \sqrt{\theta(1-\theta) / p})$, where $\mathrm{z}$ is the $\mathrm{z}$ score, $\theta$ is the study's average PAL rate, and $\mathrm{p}$ the total cases of individual surgeons. categories. As expected, we found that PAL was associated with delayed hospital discharge, an effect that persists nearly 3 weeks after surgery.

An accurate and generalizable PAL risk stratification tool could facilitate surgical decision making and patientspecific care. Some authors have advocated a fast-track discharge pathway for patients undergoing pulmonary resection to increase patient satisfaction and reduce hospital costs. $^{8,18-21}$ Given the need for carefully developed standardized protocols to effectively manage and monitor patients, the ability to predict PAL preoperatively would be valuable. In addition, both intraoperative (eg, pleural tenting, sealants, buttressed staple lines) and postoperative (eg, pneumoperitoneum, water seal, flutter valve) methods for PAL management exist, but their efficacy remains unclear. $^{10,22-27}$ For example, Cerfolio and Bryant ${ }^{18}$ showed that digital chest drainage tubes compared with analog systems reduce hospital stay and lead to quicker chest tube removal; they can also be used for remote electronic monitoring. A generalizable risk prediction tool could be used to facilitate randomized controlled trials of air leak reduction methods and help guide cost-effective use of these adjuncts.

We developed the model from patients at our institution who underwent resection for benign and malignant lung tumors operated through lobectomy/segmentectomy, wedge resection, and bilobectomy by either a thoracotomy or VATS surgical approach. Previous risk models from 
Brunelli and colleagues ${ }^{3}$ and Lee and colleagues ${ }^{1}$ excluded wedge resection and Rivera and colleagues ${ }^{2}$ included volume reduction and bullectomy. Although PAL incidence is lower after VATS wedge, inclusion of resection type and surgical approach as variables in our model allows PAL prediction for these previously, but large patient populations. Similar to Brunelli and colleagues and Lee and colleagues, we excluded lung volume reduction patients because their high rate of air leak (approaching $50 \%$ in most studies), coupled with significant differences in baseline patient characteristics (eg, higher rates of bullous emphysema) and surgical management (greater routine use of surgical adjuncts), ${ }^{25,28}$ would dominate the model and obscure the relationship between PAL and various predictors for patients with lung cancer and nodules.

The nomogram approach permitted inclusion of more risk factors plus interactions effects compared with previous work, with almost full preservation of predictive capability compared with the regression equation. A larger number of risk factors provides much greater discrimination between patients than would a simpler model that includes only a few binary variables, particularly when the groupings are common (eg, male patients with low $\% \mathrm{FEV}_{1}$ ). Consistent with previous risk factor studies, our model includes 3 commonly identified risk factors for PAL: low BMI, ${ }^{2,3}$ low $\% \mathrm{FEV}_{1},{ }^{1,3-5,9,29,30}$ and lobectomy. ${ }^{2,4,5,29}$ In addition, our population size allowed for a sufficient number of cases with PAL to include previous history of smoking ${ }^{4,9}$ and right-sided resection ${ }^{2,31}$ variables that have previously been significant on bivariable but not multivariable analysis. Annual surgeon caseload, preoperative hospitalization, reoperation, Zubrod score and the interaction effects, right-sided thoracotomy, and wedge resection by thoracotomy have not been analyzed previously. Interaction variables allow proper interpretation of risk factors; inclusion of these interactions improved the discriminatory accuracy of our model. Others have reported $\operatorname{age}^{3,31}$ and $\operatorname{sex}^{2,31}$ as independent predictors of PAL, but we found them to be nonsignificant after adjusting for the included variables. Emphysema, ${ }^{4}$ bronchitis, ${ }^{4}$ pleural adhesions, ${ }^{1-3,30}$ Deco, ${ }^{1}$ and upper lobe resection ${ }^{2,5,30}$ are other previously identified PAL risk factors, but were not available for analysis in our dataset.

Prolonged parenchymal air leaks result from impaired healing of disrupted alveoli, often associated with poor apposition of lung with parietal pleura. It is likely that surgeon- and institution-specific technical factors such as method of fissure dissection, buttressing staple lines, sealants and glues, and pleural tenting are important. ${ }^{32}$ In addition, however, reduced wound healing, increased pulmonary compliance, and inflammation could all be influential factors. ${ }^{33}$ Indeed, our finding that increasing BMI was protective against PAL has been observed by others, with underweight patients (BMI $<18.5 \mathrm{~kg} / \mathrm{m}^{2}$ ) experiencing significantly higher PAL incidence $(P<.001)$ in 1 study. ${ }^{34}$ One hypothesis is that lower BMI is a marker of lower nutritional status and poor wound healing. ${ }^{3,5,32}$ However, this does not explain the continued decrease of PAL into the overweight and obese weight classes in our study. An alternative explanation may derive from consistent findings of higher respiratory rates, lower tidal volume, reduced total respiratory system compliance, decreased expiratory reserve volume with preserved spirometry, and gas exchange and airway resistance in obese patients, ${ }^{35}$ which may produce an intrathoracic milieu that favors sealing of parenchymal defects.

It is not surprising that PAL rates differed across surgeons, because earlier studies have shown this to be an important risk factor. ${ }^{5}$ We observed higher PAL incidence with increasing caseload, and surgeon caseload remains a significant predictor after risk adjustment for case mix and patient clustering among surgeons. This finding cannot be explained by the unexpected variation from differences in baseline risk, treatment, or surgical management within the measured variables, because all surgeons fell within the $99 \%$ control limits. More likely, higher volume surgeons have different case mixes in which higher volume surgeons operate on subsets of patients with unmeasured variables contributing to higher baseline PAL risk. These unmeasured variables, not currently abstracted for the STSGTSD, could reveal modifiable technical factors to reduce the incidence of PAL and require further study. Given this, we include a more generalizable nomogram model excluding annual surgeon caseload.

\section{Strengths and Limitations}

Model development followed a step-by-step statistical analysis based on published guidelines. ${ }^{15}$ Further development requires external validation in a multicenter setting, prospective validation, and inclusion of important independent risk factors for PAL not captured in the STSGTSD (eg, pleural adhesions and segmental lung resection). We chose to keep patients with unmeasured $\% \mathrm{FEV}_{1}$, because most of these patients underwent wedge resection for a benign or secondary metastatic tumors. The unmeasured $\% \mathrm{FEV}_{1}$ category could apply to patients at other centers who similarly did not undergo preoperative lung function testing, but the finding requires external validation.

\section{CONCLUSIONS}

We have developed a clinical prediction model for PAL with good discriminatory accuracy. It has the potential to improve patient care through fast-track discharge pathways, better informed preoperative patient counseling, and selective use of surgical adjuncts. Discharge planning could 
be proactive, with patients prepared to be sent home on the second or third day with an indwelling chest tube, rather than waiting 5 or more days for the air leak to resolve. The model supports previous work analyzing risk factors for PAL while presenting a novel perspective on previously and newly identified risk factors. Prospective and external validation of our model is required to realize future clinical application.

\section{Conflicts of Interest}

Authors have nothing to disclose with regard to commercial support.

We thank Li Wang at the Clinical and Translational Science Institute for statistical support, and Connie Timko and Karan Moore for assistance with data acquisition.

\section{References}

1. Lee L, Hanley SC, Robineau C, Sirois C, Mulder DS, Ferri LE. Estimating the risk of prolonged air leak after pulmonary resection using a simple scoring system. J Am Coll Surg. 2011;212:1027-32.

2. Rivera C, Bernard A, Falcoz PE, Thomas P, Schmidt A, Bénard S, et al. Characterization and prediction of prolonged air leak after pulmonary resection: a nationwide study setting up the index of prolonged air leak. Ann Thorac Surg. 2011:92:1062-8.

3. Brunelli A, Varela G, Refai M, Jimenez MF, Pompili C, Sabbatini A, et al. A scoring system to predict the risk of prolonged air leak after lobectomy. Ann Thorac Surg. 2010;90:204-9.

4. Liang S, Ivanovic J, Gilbert S, Maziak DE, Shamji FM, Sundaresean RS, et al. Quantifying the incidence and impact of postoperative prolonged alveolar air leak after pulmonary resection. J Thorac Cardiovasc Surg. 2013;145:948-54.

5. Elsayed H, McShane J, Shackcloth M. Air leaks following pulmonary resection for lung cancer: is it a patient or surgeon related problem? Ann Royal Coll Surg Engl. 2012;94:422-7.

6. Varela G, Jimenez MF, Novoa N, Aranda JL. Estimating hospital costs attributable to prolonged air leak in pulmonary lobectomy. Eur J Cardiothorac Surg. 2005;27:329-33.

7. Okereke I, Murthy SC, Alster JM, Blackstone EH, Rice TW. Characterization and importance of air leak after lobectomy. Ann Thorac Surg. 2005;79:1167-73.

8. Brunelli A, Rocco G. Internal validation of risk models in lung resection surgery: bootstrap versus training-and-test sampling. J Thorac Cardiovasc Surg. 2006; 131:1243-7.

9. Liberman M, Muzikansky A, Wright CD, Wain JC, Donahue DM, Allan JS, et al. Incidence and risk factors of persistent air leak after major pulmonary resection and use of chemical pleurodesis. Ann Thorac Surg. 2010;89:891-7.

10. Mueller MR, Marzluf BA. The anticipation and management of air leaks and residual spaces post lung resection. J Thorac Dis. 2014;6:271-84.

11. United States Public Health Service, United States Health Care Financing Administration, National Center for Health Statistics (U.S.). ICD-9-CM international classification of diseases, ninth revision, clinical modification, sixth edition. NCHS CD-ROM 1996, no 1. Official ed. Washington, D.C.: U.S. Dept. of Health and Human Services, Public Health Service, Health Care Financing Administration; 1996.

12. Brock GN, Barnes C, Ramirez JA, Myers J. How to handle mortality when investigating length of hospital stay and time to clinical stability. BMC Med Res Methodol. 2011;11:144.

13. Austin PC, Tu JV. Bootstrap methods for developing predictive models. Am Stat. 2004:58:131-7.

14. Zlotnik A, Abraira V. A general-purpose nomogram generator for predictive logistic regression models. Stata J. 2015;15:537-46.

15. Steyerberg EW. Clinical Prediction Models: A Practical Approach to Development, Validation, and Updating. New York: Springer Science \& Business Media; 2008.
16. Harrell FE, Lee KL, Mark DB. Tutorial in biostatistics multivariable prognostic models: issues in developing models, evaluating assumptions and adequacy, and measuring and reducing errors. Stat Med. 1996;15:361-87.

17. Mayer EK, Bottle A, Rao C, Darzi AW, Athanasiou T. Funnel plots and their emerging application in surgery. Ann Surg. 2009;249:376-83.

18. Cerfolio RJ, Bryant AS. The benefits of continuous and digital air leak assessment after elective pulmonary resection: a prospective study. Ann Thorac Surg. 2008;86:396-401.

19. McKenna RJ Jr, Mahtabifard A, Pickens A, Kusuanco D, Fuller CB Fast-tracking after video-assisted thoracoscopic surgery lobectomy, segmentectomy, and pneumonectomy. Ann Thorac Surg. 2007;84:1663-7; discussion 1667-8.

20. Muehling BM, Halter GL, Schelzig H, Meierhenrich R, Steffen P Sunder-Plassmann L, et al. Reduction of postoperative pulmonary complications after lung surgery using a fast track clinical pathway. Eur J Cardiothorac Surg. 2008;34:174-80.

21. Bryant AS, Cerfolio RJ. The influence of preoperative risk stratification on fast-tracking patients after pulmonary resection. Thorac Surg Clin. 2008;18: 113-8.

22. Belda-Sanchis J, Serra-Mitjans M, Iglesias Sentis M, Rami R. Surgical sealan for preventing air leaks after pulmonary resections in patients with lung cancer. Cochrane Database Syst Rev. 2010;1:1-31.

23. De Leyn P, Muller MR, Oosterhuis JW, Schmid T, Choong CK, Weder W, et al Prospective European multicenter randomized trial of PleuraSeal for control of air leaks after elective pulmonary resection. J Thorac Cardiovasc Surg. 2011; 141:881-7.

24. Gilbert S, McGuire AL, Maghera S, Sundaresan SR, Seely AJ, Maziak DE, et al. Randomized trial of digital versus analog pleural drainage in patients with or without a pulmonary air leak after lung resection. J Thorac Cardiovasc Surg. 2015; 150:1243-9.

25. Mahajan AK, Doeing DC, Hogarth DK. Isolation of persistent air leaks and placement of intrabronchial valves. J Thorac Cardiovasc Surg. 2013;145 626-30.

26. Ng T, Ryder BA, Machan JT, Cioffi WG. Decreasing the incidence of prolonged air leak after right upper lobectomy with the anterior fissureless technique. J Thorac Cardiovasc Surg. 2010;139:1007-11.

27. Schuchert MJ, Abbas G, Landreneau JP, Luketich JD, Landreneau RJ. Use of energy-based oagulative fusion technology and lung sealants during anatomic lung resection. J Thorac Cardiovasc Surg. 2012;144:S48-51.

28. DeCamp MM, Blackstone EH, Naunheim KS, Krasna MJ, Wood DE, Meli YM et al. Patient and surgical factors influencing air leak after lung volume reduction surgery: lessons learned from the National Emphysema Treatment Trial. Ann Thorac Surg. 2006;82:197-206.

29. Cerfolio RJ, Bass CS, Pask AH, Katholi CR. Predictors and treatment of persistent air leaks. Ann Thorac Surg. 2002;73:1727-30.

30. Brunelli A, Monteverde M, Borri A, Salati M, Marasco RD, Fianchini A. Predictors of prolonged air leak after pulmonary lobectomy. Ann Thorac Surg. 2004; 77:1205-10.

31. Petrella F, Rizzo S, Radice D, Borri A, Galetta D, Gasparri R, et al Predicting prolonged air leak after standard pulmonary lobectomy: computed tomography assessment and risk factors stratification. Surgeon. 2011;9:72-7.

32. Hunt BM, Aye RW. Prolonged air leak after lung resection. Curr Resp Med Rev 2012;8:280-4

33. Arslantas MK, Kara HV, Tuncer BB, Yidizeli B, Yuksel M, Bostanci K, et al Effect of the amount of intraoperative fluid administration on postoperative pulmonary complications following anatomic lung resections. J Thorac Cardiovasc Surg. 2015;149:314-20. 321.e1.

34. Thomas PA, Berbis J, Falcoz PE, Le Pimpec-Barthes F, Bernard A, Jougon J, et al. National perioperative outcomes of pulmonary lobectomy for cancer: the influence of nutritional status. Eur J Cardiothorac Surg. 2014;45:652-9; discussion 659.

35. Littleton SW. Impact of obesity on respiratory function. Respirology. 2012;17: 43-9.

Key Words: prolonged air leak, persistent air leak, air leak, pulmonary resection, lung cancer, multivariable, risk factors, risk stratification, funnel plot 


\section{APPENDIX E1. RISK-ADJUSTED MODELS FOR SURGEON ANNUAL CASELOAD EFFECT}

Unadjusted case volume as a metric for surgical expertise is a crude measure that does not account for the variations in patient population between surgeons. In our prediction model, we adjust for the key predictors of prolonged air leak (PAL), which should account for much of the variation affecting PAL risk, but still find significantly higher risk of PAL with higher annual caseload. The relationship we see is opposite of that expected, in which higher caseload should theoretically result in superior outcome. In the surgical literature, volume-outcome relationships when observed usually occur with technically complicated procedures. For PAL after pulmonary resection, we believe unmeasured differences in baseline PAL risk and process of care both may play a role.

\section{Adjusting for Case Mix}

We created a parsimonious clinical prediction model filtering from a larger set of variables available in our institutional Society of Thoracic Surgeons (STS) database. We have the ability to risk-adjust for the additional variables we excluded as well as other differences in case mix that could affect baseline risk. In Table E1, we show that even after logistic regression adjustment for additional variables affecting case mix (variables listed in footnote), annual surgeon caseload remains a significant predictor with minimal change to the effect size, and its statistical significance.

\section{Adjusting for Clustering}

Panageas and colleagues ${ }^{\mathrm{E} 1}$ in an article on the "Clustering of Outcomes in Volume-Outcome Studies," discusses the importance of adjusting for clustering of outcomes among surgeons in studies of major medical procedures. The purpose of adjustment for clustering is to account for differences in process of care among surgeons, such as variation in surgical technique or skill, which can exaggerate the difference in the rate of PAL. In Table E1 and Figure E2 we show that after adjusting for clustering and case mix, though the size of the confidence interval is broadened and the statistical significance is somewhat attenuated, greater annual surgeon caseload remains significantly associated with increased odds of PAL. The attenuation of the effect size suggests possible differences in process of care.

\section{CONCLUSIONS}

Our additional analyses are preliminary and limited because of the extent of STS data availability. We are unable to account for unmeasured factors that we believe may be important. For example, higher volume surgeons may perform more segmentectomy (lobectomies/segmentectomy were grouped together), or differences could exist in intraoperative/postoperative management (use of preventative PAL surgical adjuncts or water seal versus suction preference). We did not account for hospital case volume, which could be a confounder because of the inability to distinguish between cases performed at 2 of our largest hospitals. Similarly, we do not adjust for clustering of PAL within hospital, which requires more complicated statistical adjustments using multilevel clustering models, given the high correlation between surgeon and hospital. At our institution, the PAL surgeon-volume effect deserves further investigation.

\section{E-Reference}

E1. Panageas KS, Schrag D, Riedel E, Bach PB, Begg CB. The effect of clustering of outcomes on the association of procedure volume and surgical outcomes. Ann Intern Med. 2003;139:658-65. 


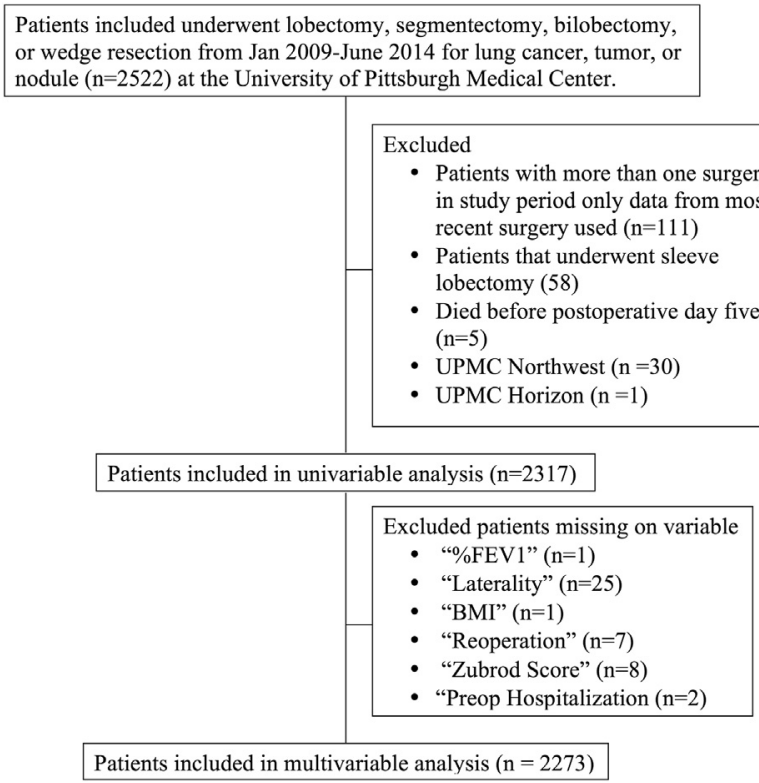

FIGURE E1. Consort diagram showing the study patient inclusion and exclusion algorithm.

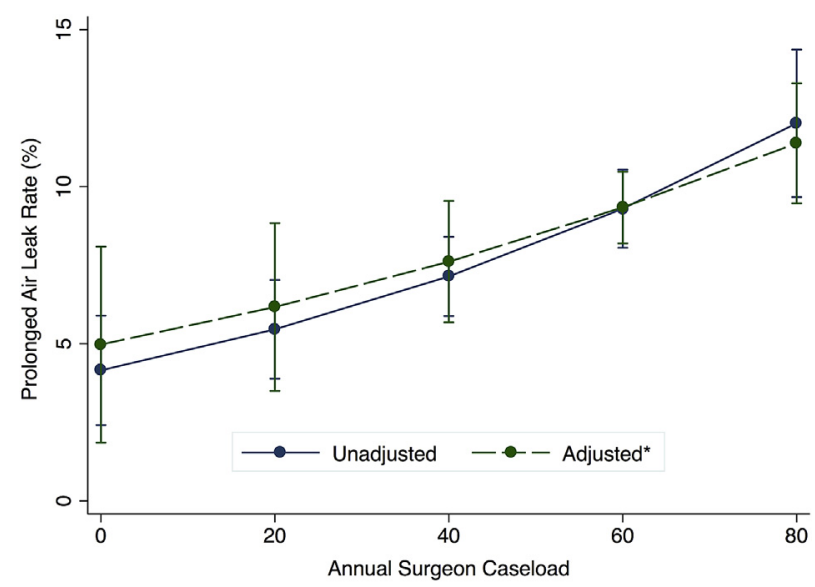

FIGURE E2. Predicted rate of prolonged air leak $(P A L)$ for unadjusted and adjusted logistic regression models. *Adjusted for PAL predictors, case mix, and clustering among surgeon as detailed in Table E1.

TABLE E1. Relationship of annual surgeon caseload to PAL occurrence after pulmonary resection

Odds ratio of PAL per

increase in annual surgeon

Volume in 20-unit case

intervals $(95 \% \mathrm{CI})$
Adjusted for predictors

in final model

Unadjusted

$1.333(1.153-1.540)$

$<.001$
Adjusted for predictors and case mix*

$274(1.089-1.491)$

Adjusted for predictors, case mix, and clustering of PAL within surgeon 1.274 (1.029-1.578)

$$
P
$$

$P A L$, Prolonged air leak; $C I$, confidence interval. *Multivariable logistic regression for all variables of the initial full model (shown in Table 2 of paper, excluding interaction effects), and race, surgery year, hospital type, ASA classification, preoperative chemotherapy, preoperative radiation therapy, previous computed tomography surgery, last hemoglobin, last creatinine, and status (variables shown in Table 1 of paper). $\dagger$ Standard error of odds ratio adjusted using clustered robust standard errors with logistic regression (generalized estimating equations method of cluster adjustment as described by Panageas and colleagues ${ }^{\mathrm{E} 1}$ with exchangeable covariate structure showed minimal correlation $<.001$, and robust standards errors minimally different from those obtained with standard logistic regression). 